\title{
EFFECT OF ACUTE L-CARNITINE SUPPLEMENTATION ON BLOOD LACTATE, GLUCOSE, AEROBIC AND ANAEROBIC PERFORMANCE IN ELITE MALE ARTISTIC GYMNASTS
}

\author{
Hamid Arazi ${ }^{1}$, Mohammad Mehrtash ${ }^{2}$ \\ University of Guilan', Rasht, Iran \\ University of Shiraz', Shiraz, Iran
}

\begin{abstract}
Background. Probably L-Carnitine can induce the increase of Pyruvate dehydrogenase activity, decrease of lactic acid production and performance improvements due to the reinforcement of long chain fatty acid oxidation and stabilize of coASH to free COA. Based on this, the aim of our study was to investigate the effects of acute L-Carnitine supplementation on blood lactate, glucose, $\mathrm{VO}_{2} \mathrm{max}$ and anaerobic power in elite male artistic gymnasts.

Methods. Eighteen male artistic gymnasts were randomly assigned to supplementation $(\mathrm{n}=9)$ or placebo $(\mathrm{n}=9)$ groups. In a double blind design, subjects participated in two tests with a break of one week between them. Subjects ingested 3 grams of L-Carnitine or placebo (maltodextrin) 90 minutes before aerobic and anaerobic exercise protocols. We used a $20 \mathrm{~m}$ shuttle run as an aerobic exercise protocol and running based anaerobic sprint test (RAST) as an anaerobic exercise protocol. Blood samples were collected 5 minutes pre and 4 minutes post-tests for the analysis of lactate and glucose.

Results. L-Carnitine group had significantly lower lactate concentration than placebo group after tests $(p<.05)$. L-Carnitine group had significantly higher blood glucose $(p<.05)$ compared with placebo group, too. $\mathrm{Also} \mathrm{VO}_{2} \mathrm{max}$ as well as mean and maximum power in L-Carnitine group were significantly higher than those in placebo group $(p<.05)$.

Conclusions. These findings indicated that acute oral supplementation of L-Carnitine can probably induce fatigue decrease and improvement of aerobic and anaerobic performance in elite male artistic gymnasts.
\end{abstract}

Keywords: L-carnitine, lactate, glucose, aerobic exercise, anaerobic exercise, gymnastics.

\section{INTRODUCTION}

1 Today, L-Carnitine is introduced as an enhancer of athletic performance (Karlic \& Lohninger, 2004). In the field of L-carnitine supplementation it has been reported that consuming this supplement can increase fat oxidation, decrease carbohydrate oxidation and improve performance (Dehghani, Shakerian, Nasseri, Nikbakht, \& Nejad, 2013), maintain the integrity of the cell membrane, establishing the physiological ratio of CoA (COA) to acetyl-CoA (coA SH) within the mitochondria, then leads to increased activity of pyruvate dehydrogenase enzyme and finally reduces lactate production (Reed, 2013; Stephens, ConstantinTeodosiu, \& Greenhaff, 2007).

On the other hand, the main substrate in anaerobic activity is carbohydrate and the pyruvate converted to lactate by lactate dehydrogenase, which affects performance (Bangsbo, Mohr, Poulsen, Perez-Gomez, \& Krustrup, 2006). Also, the production of acyl-carnitine through the buffering of CoA storage can be useful for cell function (Furuichi, 2016). High-intensity exercise is associated with hypoxia and increased concentrations of blood ammonia (ZhanQi, 2011). 
So, the availability of carnitine even in the baseline level can reduce the rate of loss of physical function and prevent muscle fatigue (Naclerio, LarumbeZabala, Cooper, Allgrove, \& Earnest, 2015; Pandareesh \& Anand, 2013; Sato et al., 2015).

Eizadi et al. (2009) investigated the Influence of short-term L-carnitine and heparin 90 minutes before exercise. The results of this study showed that short-term use of L-carnitine and heparin did not have any effect on glucose and blood lactate (Mojtaba, Maryam, Davood, \& Fatemeh, 2009). Arazi and colleagues (2013) showed that short-term supplementation of L-carnitine can reduce lactate concentration after a high intensity exercise (Arazi, Rahmaninia, Azali, \& Mehrtash, 2013). Nourshahi and Ebrahimi (2010) observed that taking 2 grams of L-carnitine before a maximum aerobic activity ( 90 minutes) reduced lactate production and increased $\mathrm{VO}_{2}$ max. Stuessi and colleagues (2005) studied the effect of $2 \mathrm{~g}$ L-carnitine supplementation before ergometer bike. Finally, results showed no significant change in the lactate concentration in post-test compared to pre-test (Stuessi, Hofer, Meier, \& Boutellier, 2005). Also, Jacobs et al. (2009) investigated the effects of shortterm 4.5 grams L-carnitine with glycine propionyl supplementation 90 minutes before the Wingate test. They found a significant reduction in blood lactate and also a significant increase in peak power of experimental group compared to the placebo (Jacobs, Goldstein, Blackburn, Orem, \& Hughes, 2009). Barnett et al. (1994) studied the effect of carnitine supplementation on lactate concentration after 14 days of high-intensity exercise. Their results did not show any effect of carnitine on lactate concentration (Barnett et al., 1994). Eroğlu et al. (2008) showed that ingestion of L-carnitine (2 grams) for 1 hour before playing badminton did not have any impact on blood lactate concentration (Eroğlu, Senel, \& Güzel, 2008). Nasuri (2014) reported that consuming 3 grams of L-carnitine 90 minutes before aerobic exercise can reduce lactate production and increase aerobic performance.

As carnitine improvise performance and reduces fatigue in the short-term and intense activity (anaerobic), it is expected that these supplements can play an ergogenic role in anaerobic activities such as gymnastics (Caine, Russell, \& Lim, 2013; Jeukendrup, 2002). Trappe et al. (1994) examined the effect of L-carnitine $(2 \mathrm{~g})$ in 100 meters speed swimming and there was no significant change in performance and lactate concentration (Trappe, Costill, Goodpaster, Vukovich, \& Fink, 1994).
Havanloo et al. showed that consumption of $30 \mathrm{mg} /$ $\mathrm{kg} / \mathrm{d}$ L-carnetine plus Q10 can improve anaerobic capacity and reduce fatigue index (Hovanloo, Nourshahi, Amini, \& Sahami, 2012).

On the other hand, since male artistic gymnasts have very severe workout, athletes who have higher aerobic fitness have a shorter recovery time and continue their workouts with high quality (Vass, 2004). In this context, Arazi and colleagues (2013) reported that consumption of L-carnitine can reduce lactate production, increase plasma glucose, $\mathrm{VO}_{2}$ max, peak and maximal power in trained men (Arazi et al., 2013).

Therefore, studies are scarce and controversial. A few studies have shown the positive effects of this supplement in anaerobic activities, and expected this supplement to have a significant role in the development of aerobic and anaerobic capacity of male artistic gymnasts. Thus, in this study we tried to investigate the effect of short-term consumption of L-carnitine supplementation on plasma glucose and lactate concentration, aerobic and anaerobic capacity in elite male artistic gymnasts.

\section{METHODS}

Subjects. This was a double-blind placebocontrolled randomized study. Considering the small number of professional male artistic gymnasts in Iran, statistical sample of this study were 18 elite male artistic gymnasts who were purposefully selected as an available sample. All subjects had experience in professional gymnastics training for more than 10 years (5-6 sessions per week) and were invited to national camp. Subjects announced their readiness by completing the consent form and health questionnaire after full awareness of the benefits and risks of the test. Seven days before the main test, subjects' anthropometric characteristics (age, height, weight, and body fat percentage) were measured to report in this study (Table 1). 3-site Jackson-Pollack equation was used for the estimation of body fat percentage. Then, the subjects were randomly divided into 2 groups ( $n$ $=9$ ) of placebo or supplementation. To eliminate individual differences, each group (placebo or supplementation) equally were bound to take the aerobic and anaerobic tests. The subjects were asked to maintain their usual diet. Based on this, the subjects were controlled for food and eating foods containing carnitine, such as meat and dairy products, for about 48 hours before the test. Also, 
in this period participants were asked to avoid severe physical activity.

Table 1. Descriptive characteristics (mean $\pm \boldsymbol{S D}$ )

\begin{tabular}{|l|l|l|}
\hline \multicolumn{1}{|c|}{ Characteristics } & \multicolumn{1}{c|}{ Mean } & \multicolumn{1}{c|}{ SD } \\
\hline Age $($ years $)$ & 21 & 2.12 \\
\hline Height $(\mathrm{cm})$ & 171.77 & 2.63 \\
\hline Weight $(\mathrm{kg})$ & 64.11 & 3.68 \\
\hline Body mass index $\left(\mathrm{kg} / \mathrm{m}^{2}\right)$ & 21.73 & 1.36 \\
\hline Body fat $(\%)$ & 10.77 & 2.63 \\
\hline
\end{tabular}

Procedure. 90 minutes before the beginning of the test participants consumed 3 grams of L-carnitine or placebo (maltodextrin) with $200 \mathrm{ml}$ of water (Jacobs et al., 2009). For aerobic and anaerobic testing the $20 \mathrm{~m}$ shuttle run (beep test), and the RAST test were used respectively (Arazi et al., 2013). $\mathrm{VO}_{2} \max$ was estimated applying the formula: $18.043461+(0.3689295 \times$ ST $)+$ $(-0.000349 \times$ ST $\times$ ST $),($ ST $=$ total number of shuttles). Also, in the part of anaerobic activity, the maximum power was equal to the highest value in RAST test, and the mean power equivalent - to the sum of all six values $\div 6$ (Mackenzie, 2005). Blood samples $(5 \mathrm{ml})$ were collected at 5 minute pre and 4 minute post-tests from the brachial vein of subjects by a technician and then lactate and glucose were analysed in the biochemistry laboratory.

Statistical Analysis. In this study, Kolomogorov-Smirnov test was used for normal distribution of the data and parametric test was used to ensure to normality of the data. Independent $t$-test was used to compare the differences between the measured variables in placebo and supplementation groups. The significance level for all tests was considered .05. Also, SPSS (version 18) software was used for all statistical procedures.

\section{RESULTS}

Mean values of body mass index and fat percentage (Jackson/Pollock 3-Site Caliper Method) of subjects were $21.73 \pm 1.36 \%$ and $10.77 \pm$ $2.63 \mathrm{~kg} / \mathrm{m}^{2}$ respectively (Table 1 ). The values of $\mathrm{VO}_{2} \mathrm{max}$, maximum and mean powers were shown in Table 2. Lactate concentration was significantly lower in the supplementation group after aerobic $(140.4 \pm 15.5 \mathrm{mg} / \mathrm{dl})$ and anaerobic $(147.8 \pm 12.8 \mathrm{mg} /$ dl) exercise compared to the placebo group (respectively $150.1 \pm 12.1$ and $157.4 \pm 10.1 \mathrm{mg} / \mathrm{dl}$ ) $(p \leq .05)$ (Figure).
Table 2. VOmax, maximum and mean power $($ mean $\pm S D)$

Note. * - Significant diffe-rence compared with placebo group at $p \leq .05$.

\begin{tabular}{|l|c|c|c|c|}
\hline \multicolumn{1}{|c|}{ Gariable } & Supplement & Placebo & $\boldsymbol{t}$ & sig \\
\hline $\mathrm{VO}_{2} \mathrm{max}(\mathrm{ml} / \mathrm{kg} / \mathrm{min})$ & $* 4.0 \pm 49.5$ & $4.8 \pm 47.6$ & -2.5 & .03 \\
\hline Maximum power (watt) & $* 77.3 \pm 486.5$ & $67.8 \pm 475.3$ & -2.4 & .04 \\
\hline Mean power (watt) & $73.0 \pm 383.6$ & $60.6 \pm 363.4$ & -2.1 & .06 \\
\hline
\end{tabular}

Figure. Comparison of plasma glucose and lactate in the placebo and supplementation groups after aerobic and anaerobic activity

Note. * - significant difference compared with placebo group at $p \leq .05$.

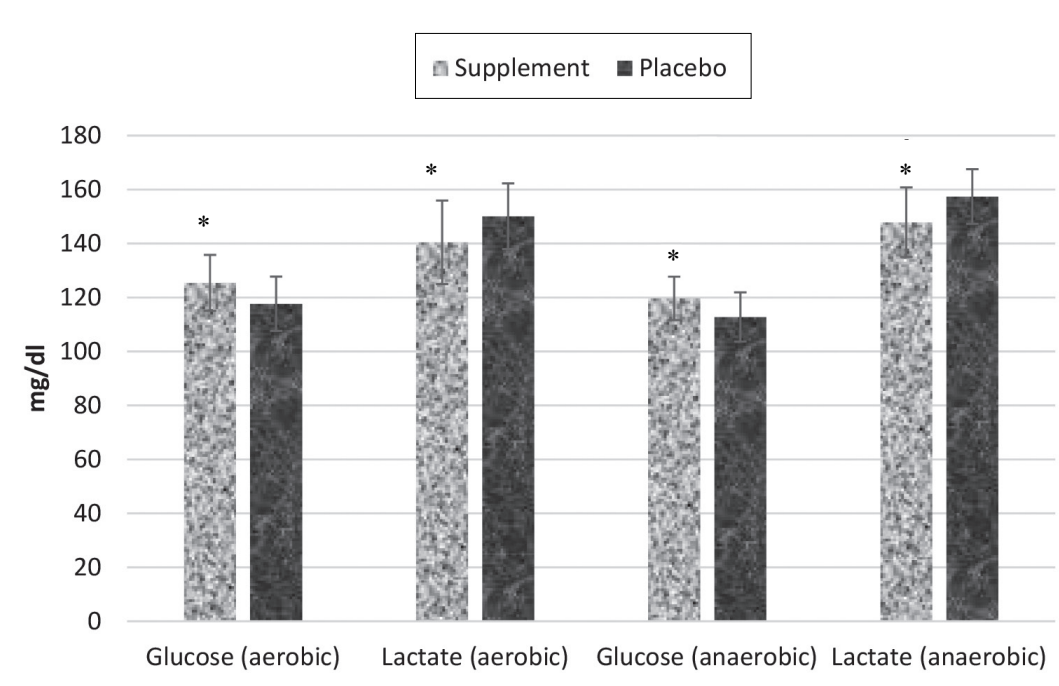


Blood glucose concentration was statistically higher after aerobic and anaerobic exercise in supplementation group (respectively $125.4 \pm 10.3$ and $119.6 \pm 8.0 \mathrm{mg} / \mathrm{dl}$ ) compared with the placebo group (respectively $117.6 \pm 10.0$ and $112.7 \pm$ $9.1 \mathrm{mg} / \mathrm{dl})(p \leq .05)$ (Figure).

\section{DISCUSSION}

L-carnitine plays a significant role in transfering long-chain fatty acids into the mitochondria through membrane. Thereby, it leads to the increase of fatty oxidation and glycogen storage. High intensity exercise can produce acetyl-CoA and carnitine that can react with it and produce acetyl L-carnitine that leads to a decrease of acetyl-CoA to CoA ratio. Also, it may increase the activity of the pyruvate dehydrogenase and cause decreasing lactate concentration (Karlic \& Lohninger, 2004). Anaerobic power is one of the success factors in the short and fast sport events such as gymnastics, and it improves when the body becomes fatigued later. Lactic acid is fatigue factor in anaerobic activity, which produces lactic acid. During intense activities, conversion of acetyl-CoA to free $\mathrm{CoA}$ ratio increases; thereby it leads to increased lactate accumulation. L-carnitine plus acetyl-CoA form acetyl L-carnitine and cause fixing this ratio that leads to reduced lactate accumulation.

Also,Krebscycleproducessomefreecoenzymes that can improve anaerobic performance. Results of our study showed that male artistic gymnasts who were consuming supplements had higher peak power and no significant difference in mean power compared to placebo group. The results of our study are in line with the ones by Jacobs and colleagues $(2010,2009)$, probably due to the dose and duration of supplementation and type of exercise (Jacobs \& Goldstein, 2010; Jacobs et al., 2009). Arazi et al (2013) showed that consuming 3 grams of L-carnitine 90 minutes before anaerobic exercise can increase peak power and mean power (Arazi et al., 2013). Also, Havanloo et al., 2012, found that carnitine supplementation can improve anaerobic capacity, but they used Q10 supplement plus carnitine that might have affected their results (Hovanloo et al., 2012). But in Trappe et al. (1994) research, no significant difference was observed in swimmers (Trappe et al., 1994). The test in Trappe study was the fast swimming with 2 min breaks between each trial. Accordingly, the difference in the type of exercise and rest time between each exercise can be due to this disruption, although the difference in the type of environments (ambient air and liquid water) can also affect the test. As gymnastics exercises need high power (Caine et al., 2013; Jeukendrup, 2002), it is likely that carnitine supplementation may improve athletic performance by increasing the male artistic gymnasts' power.

Gymnastics includes high intensity exercise and this contributes to an increase in lactate production, which can affect male artistic gymnasts' performance (Caine et al., 2013; Jeukendrup, 2002). Due to the effects of L-carnitine supplementation on the stability of acetyl-CoA to free CoA ratio and prevention of the accumulation of lactate, our results in relation to both aerobic and anaerobic exercise showed that after exercise supplementation group had lower lactate concentration compared to the placebo group. Results of our study were consistent with some previous studies (Jacobs \& Goldstein, 2010; Jacobs et al., 2009). Arazi and colleagues (2013) reported that short-term consumption of carnitine before activity can decrease lactate production and improve athletic performance after aerobic and anaerobic exercise (Arazi et al., 2013). But, Eizadi et al. (2009) did not observe significant differences in lactate concentrations. Presumably, the overlap effects of L-tartrate plus heparin could interfere with the effects of L-carnitine alone. Stuessi et al. (2005) suggested that L-carnitine supplementation had no effect on blood lactate concentration. Exercise reduces the muscle carnitine content and thereby increases the absorption of carnitine in muscle. In Stuessi study the subjects were sedentary and could not have enough carnitine in muscles and this amount is not required to show the effect of this supplement.

During activity with constant speed, increase or decrease in plasma glucose concentration is normal. In general, light activities do not have specific effect on plasma glucose. If the training load is moderate or severe, at first glucose increases and if the physical activity continues for a longer time, glucose concentration decreases and reaches lower levels than baseline (Murthy \& Pande, 1987). On the other hand, the increase in muscle carnitine leads to an increase in Triacyl glycerol and fatty acid oxidation in muscle during endurance exercise and reduces liver glycogen breakdown that leads to lower blood glucose levels (Brass, Hoppel, \& Hiatt, 1994; Rebouche, Lombard, \& Chenard, 1993). In this study, we observed that blood glucose level in supplementation group was higher than the one in placebo group after aerobic and anaerobic exercise. It could be due to the storage of glycogen and glucose 
in aerobic exercise, but increase in blood glucose after anaerobic exercise is due to the multitude of uncontrolled factors that are not clear. These results are consistent with the findings of Arazi and colleagues (2013) because of the homology of supplementation (Arazi et al., 2013). Stephens et al. (2007) stated that L-carnitine supplementation leads to reduced muscle glycolysis, increased fat oxidation, increased blood glucose concentrations and increased time to exhaustion. After L-carnitine supplementation, Eizadi and colleagues did not find any significant change in glucose levels after exercise (Eizadi et al., 2009; Mojtaba et al., 2009). The subjects of this study were sedentary persons and, considering the effect of exercise on muscle carnitine uptake, the subjects' muscles did not absorb L-carnitine and did not get the required amount of impact on fat oxidation.

Entering long-chain fatty acids into the mitochondria and to the oxidation process needs to have a carrier. L-Carnitine plays a carrier role in the transportation of fatty acids. Since fat oxidation requires oxygen more than carbohydrates, cardiovascular system should bring more oxygen to muscles. Also, L-carnitine stimulates pyruvate dehydrogenase complex and increases the entry of pyruvate into the beta-oxidation pathway, which leads to increased oxygen consumption (Karlic $\&$ Lohninger, 2004). The results of our research showed that subjects who had taken supplements had higher $\mathrm{VO}_{2}$ max compared to placebo group. These results were in line with research by Arazi et al. (2013) and Noorshahi and colleagues (2009). However, similar results were not observed by Eizadi et al (Eizadi et al., 2009; Mojtaba et al., 2009). This may be due to long-term ingestion of L-carnitine that is likely to be associated with adaptations. On the other hand, considering that half-life of L-carnitine in the body is 2 to 3 hours, so high accumulation leads to removing excess amounts through urine (Murthy \& Pande, 1987). Wachter et al. (2002) reported long-term L-carnitine supplementation without investigating the effect of it on $\mathrm{VO}_{2}$ max. Possibly long-term ( 3 months with $4 \mathrm{~g}$ daily) L-carnitine supplementation did not increase muscle carnitine content and did not show results in incremental $\mathrm{VO}_{2} \mathrm{max}$. Those having higher aerobic capacity need shorter recovery period and their energy reserves reconstruct faster (Trappe et al., 1994). However, due to the high intensity training sessions and gymnastics training (Caine et al., 2013; Jeukendrup, 2002), increasing aerobic capacity can improve male artistic gymnasts' performance in training.

\section{CONCLUSION}

We can conclude that L-Carnitine supplementation (3 g 90 minutes before exercise) can decrease production of lactic acid, increase blood glucose and improve aerobic and anaerobic performance of elite male artistic gymnasts. Finally, it can be suggested that investigations are needed with orientation to different doses of this supplement in gymnastic movements and various sub-disciplines such as aerobics, rhythmic and acrobatic gymnastics.

Acknowledgements. Authors would like to express gratitude to all the gymnasts and those who helped in this study.

Conflict of interests. Authors declare that there are no conflicts of interests regarding this research.

\section{REFERENCES}

Arazi, H., Rahmaninia, F., Azali, K., \& Mehrtash, M. (2013). The effect of acute L-Carnitine supplementation on the blood lactate, glucose, $\mathrm{VO}_{2} \mathrm{max}$ and power in trained men: A brief report. Tehran University of Medical Sciences, 71(1).

Bangsbo, J., Mohr, M., Poulsen, A., Perez-Gomez, J., \& Krustrup, P. (2006). Training and testing the elite athlete. Journal of Exercise Science \& Fitness, 4(1), 1-14.

Barnett, C., Costill, D. L., Vukovich, M. D., Cole, K. J., Goodpaster, B. H., Trappe, S. W., \& Fink, W. J. (1994). Effect of L-carnitine supplementation on muscle and blood carnitine content and lactate accumulation during high-intensity sprint cycling. International Journal of Sport Nutrition, 4(3), 280-288.

Brass, E. P., Hoppel, C. L., \& Hiatt, W. R. (1994). Effect of intravenous L-carnitine on carnitine homeostasis and fuel metabolism during exercise in humans. Clinical Pharmacology \& Therapeutics, 55(6), 681-692.

Caine, D. J., Russell, K., \& Lim, L. (2013). Handbook of sports medicine and science, gymnastics. John Wiley $\&$ Sons.

Dehghani, M., Shakerian, S., Nasseri, M. K. G., Nikbakht, M., \& Nejad, S. H. (2013). Effects of acute consumption of L-Carnitine Tartrate (LCLT) following an exhaustive aerobic exercise on serum lipoproteins levels in Iranian elite wrestlers. American Journal of Sports Science and Medicine, 1(4), 59-62.

Eizadi, M., Eghdami, A., Khorshidi, D., Doali, H., \& Kiani, F. (2009). The effect of L-carnitine and heparin supplementation on plasma glucose and lactate concentration during exercise. Journal of Rafsanjan University of Medical Sciences, 8(4), 263-272. 
Eroğlu, H., Senel, O., \& Güzel, N. A. (2008). Effects of acute L-carnitine intake on metabolic and blood lactate levels of elite badminton players. Neuro Endocrinology Letters, 29(2), 261-266.

Furuichi, Y. (2016). Mechanism of skeletal muscle contraction: Role of mechanical muscle contraction. In Glucose homeostasis musculoskeletal disease associated with diabetes mellitus (pp. 155-169). Springer.

Hovanloo, F., Nourshahi, M., Amini, E., \& Sahami, M. (2012). Effect of short term supplementation with L-carnitin and coenzyme Q10 on aerobic and anaerobic exercise performance in sedentary college men. Pajoohandeh Journal, 17(1), 8-17.

Jacobs, P. L., Goldstein, E. R., Blackburn, W., Orem, I., \& Hughes, J. J. (2009). Glycine propionyl-L-carnitine produces enhanced anaerobic work capacity with reduced lactate accumulation in resistance trained males. Journal of the International Society of Sports Nutrition, 6(1), 1. doi: 10.1186/1550-2783-6-9

Jacobs, P. L., \& Goldstein, E. R. (2010). Longterm glycine propionyl-l-carnitine supplemention and paradoxical effects on repeated anaerobic sprint performance. Journal of the International Society of Sports Nutrition, 7(1), 1. doi: 10.1186/1550-2783-7-35

Jeukendrup, A. E. (2002). Regulation of fat metabolism in skeletal muscle. Annals of the New York Academy of Sciences, 967(1), 217-235.

Karlic, H., \& Lohninger, A. (2004). Supplementation of L-carnitine in athletes: Does it make sense? Nutrition, 20(7), 709-715. doi: 10.1016/j.nut.2004.04.003

Mackenzie, B. (2005). 101 Performance evaluation tests. London: Electric Word plc.

Mojtaba, I., Maryam, C., Davood, K., \& Fatemeh, K. (2009). The effect of chronic L-carnitine L-tartarate supplementation on glucose and lactate concentration and aerobic capacity. Procedia-Social and Behavioral Sciences, 1(1), 2692-2695. http://dx.doi.org/10.1016/j. sbspro.2009.01.476

Murthy, M., \& Pande, S. V. (1987). Malonyl-CoAbinding site and the overt carnitine palmitoyltransferase activity reside on the opposite sides of the outer mitochondrial membrane. Proceedings of the National Academy of Sciences, 84(2), 378-382.

Naclerio, F., Larumbe-Zabala, E., Cooper, R., Allgrove, J., \& Earnest, C. P. (2015). A multi-ingredient containing carbohydrate, proteins L-glutamine and L-carnitine attenuates fatigue perception with no effect on performance, muscle damage or immunity in soccer players. PLoS One, 10(4), e0125188. http://dx.doi. org/10.1371/journal.pone.0125188

Nasuri, R. M. (2014). Effects of L-carnitine tartrate acute consumption on free fatty acid and lactate blood and distance traveled following aerobic exhaustive exercise on treadmill in elite athlete. Asian Journal of Multidisciplinary Studies, 2(3).
Noorshahi, M. K. M., Ebrahimi, M. (2009). Effect of acute L-Carnitine supplementation on anaerobic threshold and lactate concentration excessive exercise. Nutrients Journal, 7(1), 45-52.

Pandareesh, M., \& Anand, T. (2013). Ergogenic effect of dietary L-carnitine and fat supplementation against exercise induced physical fatigue in Wistar rats. Journal of Physiology and Biochemistry, 69(4), 799-809. doi: 10.1007/s13105-013-0256-5

Rebouche, C. J., Lombard, K. A., \& Chenard, C. A. (1993). Renal adaptation to dietary carnitine in humans. The American Journal of Clinical Nutrition, 58(5), 660-665.

Reed, L. J. (2013). Regulation of mammalian pyruvate dehydrogenase complex by a phosphorylationdephosphorylation. Biological Cycles: Current Topics in Cellular Regulation, 18, 95.

Sato, F., Omura, T., Ishimaru, M., Endo, Y., Murase, H., \& Yamashita, E. (2015). Effects of daily astaxanthin and 1-carnitine supplementation for exercise-induced muscle damage in training thoroughbred horses. Journal of Equine Veterinary Science, 35(10), 836-842. http:// dx.doi.org/10.1016/j.jevs.2015.08.003

Stephens, F. B., Constantin-Teodosiu, D., \& Greenhaff, P. L. (2007). New insights concerning the role of carnitine in the regulation of fuel metabolism in skeletal muscle. The Journal of Physiology, 581(2), 431-444. https://dx.doi.org /10.1113\%2Fjphysiol.2006.125799

Stuessi, C., Hofer, P., Meier, C., \& Boutellier, U. (2005). L-Carnitine and the recovery from exhaustive endurance exercise: A randomised, double-blind, placebocontrolled trial. European Journal of Applied Physiology, 95(5-6), 431-435. https://dx.doi.org/10.1007/s00421005-0020-9

Trappe, S., Costill, D., Goodpaster, B., Vukovich, M., \& Fink, W. (1994). The effects of L-carnitine supplementation on performance during interval swimming. International Journal of Sports Medicine, 15(04), 181-185.

Vass, S. (2004). The role of aerobic fitness in recovery and performance during high intensity intermittent exercise (HIIE): Implications for the training of athletes. Retrieved from http://www. athleteconsulting.net/wpcontent/uploads/2011/02/Vass.pdf

Wächter, S., Vogt, M., Kreis, R., Boesch, C., Bigler, P., Hoppeler, H., \& Krähenbühl, S. (2002). Long-term administration of L-carnitine to humans: effect on skeletal muscle carnitine content and physical performance. Clinica Chimica Acta, 318(1), 51-61.

ZhanQi, W. (2011). Methods of weight control for rhythmic gymnastic athletes adopt L-carnitine. Paper presented at the Human Health and Biomedical Engineering (HHBE), 2011 International Conference. https://doi.org/10.1109/HHBE.2011.6029020 